Monatsschr Kinderheilkd $2021 \cdot 169: 470-471$ https://doi.org/10.1007/s00112-021-01171-3 Angenommen: 9. März 2021

Online publiziert: 8. April 2021

(C) Springer Medizin Verlag GmbH, ein Teil von Springer Nature 2021

\section{Redaktion}

R. Berner, Dresden

G. Hansen, Hannover

R. Kerbl, Leoben

F. Zepp, Mainz

\author{
Lukas Mileder $^{1,2}$. Philipp Jung ${ }^{1,3,4}$. Florian Hoffmann ${ }^{1,3,5} \cdot$ Ellen Heimberg $^{1,3,6}$ \\ ${ }^{1}$ Netzwerk Kindersimulation e. V., Tübingen, Deutschland \\ ${ }^{2}$ Klinische Abteilung für Neonatologie, Universitätsklinik für Kinder- und Jugendheilkunde, Medizinische \\ Universität Graz, Graz, Österreich \\ ${ }^{3}$ PAEDSIM e. V., Tübingen, Deutschland \\ ${ }^{4}$ Klinik für Kinder- und Jugendmedizin, Universitätsklinikum Schleswig-Holstein, Campus Lübeck, Lübeck, \\ Deutschland \\ ${ }^{5}$ Kinderklinik und Kinderpoliklinik im Dr. von Haunerschen Kinderspital, Ludwig-Maximilians-Universität, \\ Campus Innenstadt, München, Deutschland \\ ${ }^{6}$ Klinik für Kinderheilkunde und Jugendmedizin, Universitätsklinikum Tübingen, Tübingen, Deutschland
}

\title{
Gemeinsam sicher für unsere kleinen und kleinsten Patient*innen trainieren
}

Insofern stimmen wir mit unseren Kollegen vollends überein, dass Simulationstraining im Team 1) ein unverzichtbarer Bestandteil der Aus- und Weiterbildung im Gesundheitswesen ist und 2) aufgrund seines Potenzials zur Verbesserung von Handlungskompetenz und Patient ${ }^{*}$ innen-Outcome $[2,3]$ den gleichen Stellenwert wie das direkte klinische Arbeiten haben muss.

Mit großer Sorge haben wir in unseren eigenen medizinischen Tätigkeitsbereichen, aber auch als Vertreter*innen von PAEDSIM e.V. und Netzwerk Kindersimulation e.V. beobachtet, dass seit Beginn der durch die „coronavirus disease 2019“ (COVID-19) verursachten Pandemie zahlreiche simulationsbasierte Teamtrainings mit essenziellen medizinischen Inhalten aus unterschiedlichen Gründen verschoben oder gar gänzlich abgesagt wurden. Obgleich die aus dem Trainingsdefizit erwachsenden negativen Konsequenzen unsererseits nicht eindeutig abschätzbar sind, muss doch begründeterweise befürchtet werden, dass damit Qualitätseinbußen in der Behandlung von kritisch kranken Kindern und Jugendlichen einhergehen werden. Dies wollen wir als Ärzt* ${ }^{*}$ nnen, aber auch als Simulationstrainer*innen und Expert ${ }^{\star}$ innen für Patient ${ }^{\star}$ innensicherheit keinesfalls begünstigen oder auch nur zulassen!
Während wir den kritischen Blick auf unsere Stellungnahme schätzen, stellen wir jedoch fest, dass in dem Leserbrief zwei unterschiedliche Aspekte miteinander vermengt werden, namentlich die Forderung nach gesetzlich verpflichtenden Simulationstrainings im Team und die Kritik an unserer Zurückhaltung in der Forderung nach simulationsbasierten Trainings während der COVID-19-Pandemie [4]. Im Folgenden möchten wir zu beiden Aspekten Stellung beziehen.

Wir als Vertreter*innen von PAEDSIM e.V. und Netzwerk Kindersimulation e.V. teilen seit Langem den Wunsch nach gesetzlich vorgeschriebenen, regelmäßigen neonatologisch-pädiatrischen Simulationstrainings für Personal im Gesundheitswesen. In diesem Kontext empfiehlt daher auch die Arbeitsgruppe für Pädiatrische Simulation und Patient*innensicherheit der Österreichischen Gesellschaft für Kinder- und Jugendheilkunde in einem demnächst veröffentlichten Positionspapier „gesetzlich vorgeschriebene, verpflichtende Trainings von neonatologischen bzw. pädiatrischen Basisreanimationsmaßnahmen, mindestens alle sechs Monate für medizinisches und pflegerisches Personal in notfallmedizinischen Bereichen (z.B. Notaufnahme) und auf intensivmedizinischen oder intermediate-careStationen sowie zumindest jährliche 
Trainings für ärztliches und pflegerisches Personal in anderen Bereichen ... “. Diese Arbeitsgruppenempfehlung soll unseren Fachbereich der Kinder- und Jugendmedizin näher an den Standard der „high reliability organizations" [5] heranbringen und kann hierbei wegbereitend sein.

Seit der Erstellung unserer Stellungnahme [4] vor 6 Monaten hat nicht nur das medizinische Wissen über COVID19 deutlich zugenommen, sondern wir als Mitarbeiter*innen im Gesundheitswesen haben in der Zwischenzeit auch sicheres Verhalten sowie Anwendung und Einsatz von persönlicher Schutzausrüstung optimiert. Zudem gehören regelmäßige Testungen von Gesundheitspersonal auf eine Infektion mit dem „severe acute respiratory syndrome coronavirus type 2" (SARS-CoV-2) mittlerweile zum Standard, sodass wir nunmehr auf geänderte Rahmenbedingungen blicken. Aus heutiger Sicht erscheint es schwer $\mathrm{zu}$ argumentieren, warum rezent SARS-CoV-2-negativ getestete Mitarbeiter*innen im Gesundheitswesen, die unter Wahrung der erforderlichen Schutzmaßnahmen regelmäßig miteinander reale Patient ${ }^{*}$ innen betreuen, nicht auch gemeinsam an essenziellen Notfallsimulationstrainings teilnehmen sollen. Die von Jens-Christian Schwindt und Hans Härting vorgeschlagenen Maßnahmen werden helfen, das Infektionsrisiko im Rahmen von Teamtrainings weiter zu reduzieren. Aus unserer Sicht erscheinen hierzu v. a. die Vermittlung von theoretischen Inhalten mithilfe von Online-Fortbildungen zur Reduktion der direkten Kontaktzeit der Trainingsteilnehmer*innen, die Trainingsabhaltung in kleinstmöglichen, jedoch realistischen Gruppen, sowie die stringente Einhaltung erforderlicher hygienischer Sicherheitsmaßnahmen, einschließlich Hände- und Materialhygiene, Tragen von Masken der „Filtering-face-piece“(FFP)Kategorie 2 und (wo möglich) Sicherheitsabstand wesentlich. Nachdem wir jedoch nicht unseren alleinigen Fokus auf die Behandlungsqualität unserer $\mathrm{Pa}$ tient ${ }^{*}$ innen, sondern auch auf die Sicherheit des mit deren Versorgung betrauten Personals legen wollen, möchten wir betonen, dass trotz aller Schutzmaßnahmen Trainingsaktivitäten engmaschig im Hinblick auf mögliche Ansteckungsfälle observiert werden sollten, um im Bedarfsfall frühestmöglich adäquate sicherheitsoptimierende Maßnahmen einleiten zu können.

Jedoch müssen wir auch die traurige Realität anerkennen, dass Gesundheitspersonal in diesen Tagen nicht zuletzt aufgrund von temporären De-facto-Klinikschließungen durch regionale COVID-19-Ausbrüche [6] - mit der Aufrechterhaltung der Patient*innenversorgung besonders geund mitunter auch überfordert ist $[7,8]$. Dies gilt nicht zuletzt aufgrund von temporären De-facto-Klinikschließungen durch regionale COVID-19-Ausbrüche [6]. Daher kann und darf die wünschenswerte Wiederaufnahme bzw. Weiterführung von Notfallsimulationstrainings im Team nicht auf Kosten des ohnehin bereits geforderten Personals gehen. Sie soll auch nicht von enthusiastischen Einzelpersonen abhängig sein, sondern ist vielmehr ein Auftrag an Krankenanstaltenträger, Betriebsverantwortliche und politische Entscheidungsträger*innen.

\section{Korrespondenzadresse}

\section{Dr. Lukas Mileder}

Klinische Abteilung für Neonatologie, Universitätsklinik für Kinder- und Jugendheilkunde, Medizinische Universität Graz Auenbruggerplatz 34/2, $8036 \mathrm{Graz}$, Österreich lukas.mileder@medunigraz.at

\section{Einhaltung ethischer Richtlinien}

Interessenkonflikt. L. Mileder ist 1. Vorsitzender des Netzwerk Kindersimulation e. V. sowie stellvertretender Leiter des Clinical Skills Center und stellvertretender organisatorischer Leiter der postgraduellen Universitätslehrgänge "Medical Simulation" an der Medizinischen Universität Graz. Er führt für die SIMCharacters Training GmbH (Wien, Österreich) auf Honorarbasis simulationsbasierte Trainings durch. P. Jung ist 1. Vorsitzender von PAEDSIM e. V. und führt für PAEDSIM und die SIMCharacters Training GmbH (Wien, Österreich) Simulationstrainings auf Honorarbasis durch. F. Hoffmann führt als Trainer für PAEDSIM e. V. und die SIMCharacters Training GmbH (Wien, Österreich) Simulationstrainings auf Honorarbasis durch. E. Heimberg ist als Trainerin für PAEDSIM e. V. tätig und Mitglied des Beirats des Netzwerk Kindersimulation e. V. Sie führt für PAEDSIM e.V. und die SIMCharacters Training GmbH (Wien, Österreich) Simulationstrainings auf Honorarbasis durch. Ansonsten bestehen keine finanziellen Interessenkonflikte.
Dieser Beitrag beinhaltet keine Studien an Menschen oder Tieren.

\section{Literatur}

1. Schwindt JC, Härting $H$ (2021) Dringender Appell zu gesetzlich geregelten Sicherheits- und Trainingsstandards. Monatsschr Kinderheilkd. https://doi.org/10.1007/s00112-021-01170-4

2. Steiner M, Langgartner M, Cardona F, Waldhör T, Schwindt J, Haiden N, Berger A (2015) Significant reduction of catheter-associated blood stream infections in Preterm neonates after implementation of a care bundle focusing on simulation training of central line insertion. Pediatr Infect Dis J 34(11):1193-1196

3. Theilen U, Fraser L, Jones P, Leonard P, Simpson D (2017) Regular in-situ simulation training of paediatric Medical Emergency Team leads to sustained improvements in hospital response to deteriorating patients, improved outcomes in intensive care and financial savings. Resuscitation 115:61-67

4. Jung P, Mileder L, Hoffmann F, Heimberg E (2020) Simulation-based pediatric emergency team training in times of the SARS-CoV-2 pandemic. Monatsschr Kinderheilkd 168:1130-1131

5. Polonsky MS (2019) High-reliability organizations: the next frontier in healthcare quality and safety. JHealthc Manag 64(4):213-221

6. Klinikum Bayreuth Rund 3.000 Mitarbeiter unter Quarantäne. https://www.aerzteblatt.de/ nachrichten/120545/Klinikum-Bayreuth-Rund3-000-Mitarbeiter-unter-Quarantaene.Zugegriffen: 5. Febr. 2021

7. Klinikpersonal fühlt sich oft überfordert. https:// www.tagesschau.de/investigativ/br-recherche/ corona-krankenhaus-107.html. Zugegriffen: 5. Febr. 2021

8. Azoulay E, De Waele J, Ferrer R et al (2020) Symptoms of burnout in intensive care unit specialists facing the COVID-19 outbreak. Ann Intensive Care 10(1):110 